\title{
Krankheiten in der schönsten Jahreszeit Sommer - Sonne - Sonnenstich
}

\author{
U. Rendenbach, Duderstadt \\ H. Sandholzer, Abteilung für Allgemeinmedizin der Universität Leipzig
}

NOTFALLMEDIZIN 2003; 29: 282-289

Sonnenstrahlen können den menschlichen Organismus durch ihre Energie schädigen: Reaktionen der Haut, des Auges und des Immunsystems sind die Folgen. Erhöhte Ozonbelastungen im Sommer können Entzündungen der Schleimhäute verursachen, welche zu Konjunktivitis, Pharyngitis, Laryngitis und Tracheitis, manchmal kombiniert mit Kopfschmerzen, führen. Als Hitzeschäden werden allgemein die Folgen einer gestörten thermischen Homöostase bezeichnet, die unterschiedliche Auswirkungen haben können. Bei der Hitzeerschöpfung reagiert der Organismus mit wechselnden Schockzeichen. Stören hohe Temperaturen lange die Wärmeregulation, kommt es zum Hitzschlag. Starke Sonneneinstrahlung ohne Kopfbedeckung kann zu einen „Sonnenstich“ führen, der mit heftigen Kopfschmerzen, Übelkeit, Fieber, Schwindel, Ohrensausen und orthostatischem Kollaps verbunden ist. „Akuter Brechdurchfall“ bzw. Reiseoder Sommerdiarrhoe, hat an heißen Tagen seine Ursache in verdorbenen Lebensmitten durch falsche Lagerung.
$\mathrm{S}$ ommer und Sonne bedeuten für viele Menschen Urlaub und sich häufiger und länger im Freien aufzuhalten. Freizeit und veränderte, ungewohnte Aktivitäten führen zu anderen Verletzungen, Unfällen und Krankheiten als im Winter. Sonnenbaden um braun zu sein, entspricht dem heutigen Schönheitsideal und wird allzu oft übertrieben mit zum Teil erheblichen Folgen: Sonnenbrand und polymorphe Lichtdermatosen (L55 und L56). Die seit mehreren Jahren im Sommer periodisch wiederkehrende Warnung vor der Sonne, hat dies nicht geändert, fördert aber die Angst vor dem „schwarzen Krebs“, der die verängstigten Patienten in die Haut- und Hausarztpraxen treibt. Allgemein kann aber nicht nur die energiereiche Sonnenstrahlung Schäden verursachen, sondern die Hitze belastet den Organismus auch allgemein, Regulationsmechanismen können dekompensieren (T67). Nahrungsmittel „verderben“ eher, Krankheitserreger vermehren sich schneller, und können so eine Diarrhoe verursachen.

\section{Sonnenlicht}

Durch ihre Energie können Sonnenstrahlen den menschlichen Organismus schädigen: Reaktionen der Haut, des Auges und des Immunsystems sind die Folgen. Das Spektrum der auf die Erde gelangenden Sonnenstrahlung beinhaltet Infrarot (780-3000 nm), sichtbares Licht (380-780 $\mathrm{nm})$ und Ultraviolett (UVA1: 340-380 nm, UVA2: 320340 nm, UVB: 290-320 nm). Der „dermatologische Stellenwert" der einzelnen Bereiche ist unterschiedlich. So ist UVB für den Sonnenbrand und die epidermalen Veränderungen im Rahmen des chronischen Lichtschadens verantwortlich, UVA

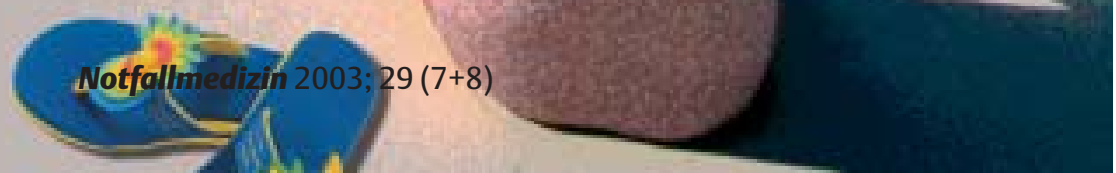


ist zu zirka 8\% an der Entstehung des Sonnenbrandes mitbeteiligt. In der Globalstrahlung (Karibik) beträgt die UVA-Energie etwa das 11 fache der UVBEnergie. Bei niedrigen Sonnenständen steigt jedoch der prozentuale Anteil der UVA-Energie an und erreicht in Europa etwa das 30fache. Als Schutz gegen UV-Strahlung besitzt die Haut eine Hornschicht und die Möglichkeit der Pigmentierung. Beide Systeme sind adaptiv, bei Belastung kommt es zu einem verstärkten Schutzeffekt. Die physiologische Reaktion der Haut ist die vermehrte Melaninbildung, aber auch eine Hyperkeratose (Lichtschwiele). Schon eine schwache Einstrahlung von Photonen (kleinste Beträge elektomagnetischer Energie bestimmter Frequenz und Wellenlänge, $\mathrm{E}=\mathrm{h} \mathrm{x} v$ ) des UVB-Bereichs kann die DNS in den Epidermiszellen verändern, bleibt die Dosis aber deutlich unter der Sonnenbrandschwelle, werden Schäden rasch durch Repair-Systeme behoben. Neben der bei der weißen Bevölkerung als schön geltenden Pigmentierung gibt es viele unerwünschte Hautveränderungen durch die Strahlenbelastung des Sonnenlichts. Sogar bei der Psoriasis vulgaris tritt in etwa 3\% der Fälle eine Verschlechterung ein. Die Immunsuppression der Sonnenbestrahlung kann zu einem „Herpes simplex solaris“ führen. Durch Strahlenbelastung werden außerdem die echten Photodermatosen wie die polymorphen Lichtdermatosen (PLE, polymorphous light eruptions) und die Porphyrinopathien ausgelöst. Schließlich gibt es noch Dermatosen, bei denen Licht provozierend und/oder krankheitsverschlimmernd wirken kann. Dazu gehören die Acne aestivalis, Albinismus, Herpes simplex, Lupus erythematodes, Pellagra, verschiedene Formen der Porphyrie und Xeroderma pigmentosum.

Unter Licht- oder auch Photodermatosen fasst man Reaktionen der Haut zusammen, die durch Lichteinwirkung entstehen. Daran ist die Ultraviolettstrahlung (UV), ein Bereich kleinerer Wellenlängen der elektromagnetischen Wellen, der dem blau-violetten Bereich des sichtba-

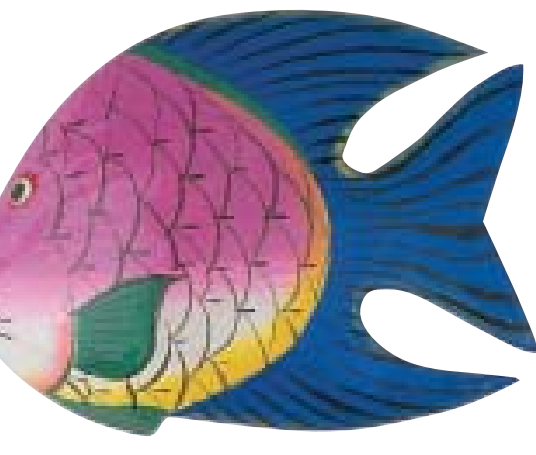

ren Lichts folgt, maßgeblich beteiligt. In der Medizin kennt man die Bräunungsstrahlung UVA - die auch Fensterglas durchdringt, was bei langen Autofahrten in der Sonne bedacht werden muss - und die erythemerzeugende UVB-Strahlung (früher Dorno-Strahlung). Die biologischen Wirkungen, zum Beispiel die Bildung von Vitamin D aus Ergosterin, und damit auch die medizinische Bedeutung ist erheblich.

\section{Dermatitis solaris -}

\section{Sonnenbrand}

Im Umgang mit der Sonne werden nicht nur im südlichen Urlaubsland jedes Jahr dieselben Fehler gemacht. Bedacht werden muss nämlich, dass das Vorbräunen im selektiv UVA-strahlenden Sonnenstudio die Haut nicht ausreichend gegen Sonnenbrand schützt. Auch ein anhaltendes Training kann zum Beispiel die individuelle Sonnenvulnerablität des eigenen Hauttyps kaum verändern, daher ist stets aufs Neue eine vorsichtige, hauttypspezifische Adaptation an ungewohnte Strahlungsintensitäten erforderlich. Als typische Sommerkrankheit erlebt der Arzt die akute Dermatitis solaris (Sonnenbrand) als phototraumatische Reaktion Gesunder durch exzessives Sonnenbaden. Das Bild gleicht der Verbrennung mit allen Entzündungszeichen bis zur Blasenbildung. Bei fortgesetzter Exposition kann eine Atrophie der Epidermis mit groben Hautreliefs, Keratosen, Pigmentflecken und dem gefürchteten Hautkrebs (Plattenepithelkarzinom und Basaliom) folgen. Es dauert etwa 18 Stunden, bis nach einer zu intensiven Sonnenexposition die Dermatitis solaris als Verbrennung I. bis II. Grades als Vollbild eines Sonnenbrands auftritt. Zusätzlich können Kopfschmerzen, Übelkeit und
Fieber auftreten. Therapeutisch lindern kühlende Umschläge den brennenden Schmerz, äußerlich können Kortikoide (etwa Jellin ${ }^{\circledR}$ Lotio) und in schwereren Fällen auch systemische Kortikoide eingesetzt werden, vornehmlich als Einmalapplikation durch den Arzt, um von vornherein jeglichen Missbrach zu verhindern (unter Kortison länger zu bräunen).

Die Rezeptur 1-3\% Hydrokortison in Oleum olivarum fettet die geschädigte Haut und mildert die Entzündung (für Kinder geeignet). Cyclooxigenasehemmer wie Acetylsalicylsäure oder - schwächer Paracetamol lindern die akuten Beschwerden.

Das sonnenbrandinduzierte Risiko für Langzeit-Hautschäden bis hin zum Hautkrebs bleibt aber ungeachtet einer solchen Akutbehandlung bestehen. Mit dem Satz „die Haut vergisst nichts“ lassen sich uneinsichtige Menschen motivieren, nicht jedes Jahr einen Sonnenbrand zu tolerieren.

\section{Polymorphe Lichtdermatose \\ (PLD)}

Während bei intensiver Sonnenexposition jeder Mensch vom Sonnenbrand bedroht ist, setzt die polymorphe Lichtdermatose eine vom Hauttyp weitgehend unabhängige endogene Bereitschaft voraus. Das Krankheitsbild kommt eher in den gemäßigten Zonen vor, entsteht durch Sonneneinwirkung und führt zu stark juckenden Hautveränderungen. Zwar ist die Ätiologie noch nicht sicher bekannt, jedoch wird als Pathogenese eine zellvermittelte im-

munologische Reaktion vom verzögerten Typ angenommen. Dafür spricht der klinische Verlauf sowie die Art der Effloreszenzen und das histopathologische Bild. Ein auslösendes Allergen wurde bislang nicht gefunden. Experimentelle Untersuchungen lassen an Hitzeschockproteine als antigenwirksame Photoprodukte denken. Die Patienten haben normale Erythem- und Pigmentierungsreaktionen. Vielleicht können Betroffene diesen - mit Juckreiz, fleckiger Rötung, Knötchen, 
Substanzen (z.B. Medikamente wie Sulfonamide) hervorgerufen werden. Die Diagnose lässt sich durch eine Epikutantestung mit anschließender Belichtung stellen. Miliaria (auch Friesel oder prickly heat) sind hirsekorngroße, wasserhelle Bläschen, die eher in den Tropen bei Kindern nach starkem Schwitzen und Verlegung der Schweißdrüsengänge auftreten können als entzündliche Reaktion der Dermis.

\section{Sonnenschutzprodukte}

Den besten Schutz stellt nach wie vor die Bedeckung der Haut mit Textilien und die Vermeidung direkter Bestrahlung dar. Die meisten Patienten lassen sich jedoch zu diesen Maßnahmen nur schwer motivieren, sie erwarten einen ausreichenden Schutz durch Lichtschutzprodukte. Die Wirkstärke wird als „Lichtschutzfaktor" angeben, so bedeutet Faktor 15, dass nach Applikation des Präparates erst eine 15fache Strahlendosis (meistens mit UVB gemessen) eine Hautrötung induziert. Derzeit sind photochemische Sonnenschutzmittel, die das UV-Licht absorbieren und physikalische Mittel, die es reflektieren, im Handel. Neuere Produkte, z.B. die Kombination von Mexoryl SX mit dem neuen Filter Mexoryl XL verbessern die UVToleranz, denn der photostabile Lichtschutzfilter hat eine Wirkung über ein breites UVA- und UVBSpektrum. Allerdings wird die Gefahr der Melanomentwicklung durch diese Stoffe nicht verringert Kinder sind besonders gefährdet. Für extreme Sonnenbelastungen (Windsurfen, Segeln oder Frühjahrsskifahren) reichen auch die stärksten Sonnenschutzprodukte nicht aus.

\section{Ozonbelastung}

Ozon ist die allotrope Modifikation des Sauerstoffs, ein dreiatomiges $\left(\mathrm{O}_{3}\right)$ blassblaues, sehr giftiges Gas mit oxidierender Wirkung und einer besseren Löslichkeit als Sauerstoff. Das atmosphärische Ozon wird in einer Höhe von zirka 20-25 km durch $\mathrm{O}_{2}$-Spaltung unter Sonnenlicht gebildet. Es absorbiert UVStrahlung und ist somit erwünscht. In Erdnähe wird Ozon durch den
Zerfall von Stickstoffdioxid $\left(\mathrm{NO}_{2}\right)$ durch Sonnenlicht gebildet, dort, wo viele Schadstoffe anfallen, zum Beispiel an Autobahnen. $\mathrm{NO}_{2}$ wird durch UV-Licht in NO und atomaren Sauerstoff gespalten. Dieser verbindet sich mit molekularem Sauerstoff der Luft zu Ozon. Allerdings ist der Prozess umkehrbar, so dass ohne UV-Strahlung (bei Regen) Ozon wieder gespalten und damit beseitigt wird. In NO-armen Gebieten (z.B. Wälder) halten sich länger höhere Ozonwerte. In den Konzentrationen in Erdnähe ist Ozon unsichtbar und geruchlos, aber reaktionsfreudig, weswegen es eine gesundheitsschädigende Wirkung entfalten kann. Die aktuellen Ozonwerte werden bekannt gegeben, beispielsweise stellt sie das Landesumweltamt ins Internet, wobei der Grenzwert mit 0,25 mg pro Kubikmeter Luft angenommen wird; wird er überschritten, kann ein allgemeines Fahrverbot verhängt werden. Der Grenzwert in der Schweiz (Quelle: Internet) liegt bei $0,12 \mathrm{mg} / \mathrm{m}^{3}$ Luft (Schweizer Luftreinhalteverordnung von März 1998). Dieser Immissionsgrenzwert wurde bei letzten Messungen allerdings oft überschritten. Die meisten Menschen sind aber eher unempfindlich gegenüber Ozonbelastungen im Sommer, etwa 85\% spüren keine Symptome, auch nicht bei Grenzwertüberschreitungen. Bei ihnen wird Ozon erst bei körperlicher Anstrengung zum Problem, weil eine Beschleunigung und Vertiefung der Atmung (Erhöhung des Atemminutenvolumens und ein vermehrter Sauerstoffverbrauch) wegen der verminderten Aufnahmekapazität der entzündeten Schleimhäute (Alveolen) schlechter möglich ist. Sportler oder Arbeiter, die im Freien tätig sind, können mit Entzündungen der Schleimhäute reagieren. Andererseits können bereits Konzentrationen von $0,2 \mathrm{mg} / \mathrm{m}^{3}$ Luft zu Reizungen der Schleimhäute führen, die zum Arzt als Konjunktivitis, Pharyngitis, Laryngitis und Tracheitis, manchmal kombiniert mit Kopfschmerzen, führen. Vorerkrankte Menschen, Asthmatiker und COPDPatienten, aber auch Säuglinge leiden eher unter Ozonbelastung. Asthmaanfälle nehmen bei Konzen- trationen von mehr als $0,24 \mathrm{mg} / \mathrm{m}^{3}$ zu, daher wurden Richtwerte festgelegt: $\mathrm{Ab}$ einer Konzentration von $0,18 \mathrm{mg} / \mathrm{m}^{3}$ sollen Kranke und gefährdete Menschen, und ab 0,36 $\mathrm{mg} / \mathrm{m}^{3}$ alle - auch Gesunde - jegliche anstrengende Tätigkeiten im Freien vermeiden. Bei Asthmatikern und bei Menschen mit hyperreagiblem Bronchialsystem führt die Schleimhautreizung zur Spastik mit erhöhtem Atmungswiderstand. Husten und Atemnot sind dann typische Beschwerden. Studien aus Gegenden mit sehr hoher Ozonbelastung (Kalifornien, Mexiko) weisen darauf hin, dass lang andauernder Kontakt mit Ozon zu permanenter Verschlechterung der Lungenleistung und chronischen Schäden der Nasenschleimhäute führen kann.

\section{Hitzeschäden}

Thermorezeptoren (z.B. KrauseEndkolben in der Haut) registrieren Temperaturänderungen, vermitteln diese an im vorderen Hypothalamus lokalisierte Areale, deren Aufgabe die Koordination der Wärmeregulation ist, und zwar nicht nur Wärmebildung und -abgabe, sondern auch Reaktionsweisen, die für den Energiehaushalt relevant sein können. Als Zusammenspiel solcher Maßnahmen resultiert die Wärmeregulation zur Erhaltung der normalen Körpertemperatur. Im Sommer bei hoher Umgebungstemperatur ist die Wärmeabgabe wesentlich, deren physikalische Komponente Strahlung, Leitung, Konvektion sowie Verdunstung von Schweiß über die Haut gestört wer-

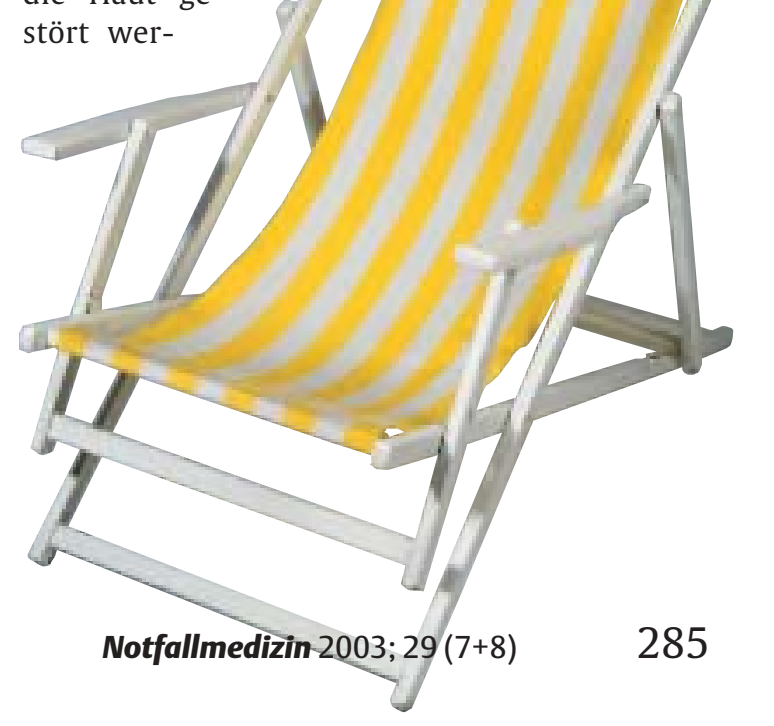


den kann und damit zu typischen „Sommerkrankheiten“ führen. Herz und Kreislauf sind dabei wesentlich beteiligt. Als Hitzeschäden werden allgemein die Folgen einer gestörten thermischen Homöostase bezeichnet, die unterschiedliche Auswirkungen haben können.

\section{Hitzeerschöpfung}

Bei der Hitzeerschöpfung reagiert der Organismus mit wechselnden Schockzeichen, weil die Thermoregulation vorrangig durch Schwitzen und verstärkte Herzarbeit gewährleistet wird. Dehydratation und Elektrolytverlust ohne ausreichende Flüssigkeitszufuhr erklären die Symptomatik - eine deutliche Erhöhung der Körpertemperatur tritt (noch) nicht auf. Nach erheblicher Anstrengung, zum Beispiel bei Märschen in der Truppe, ist

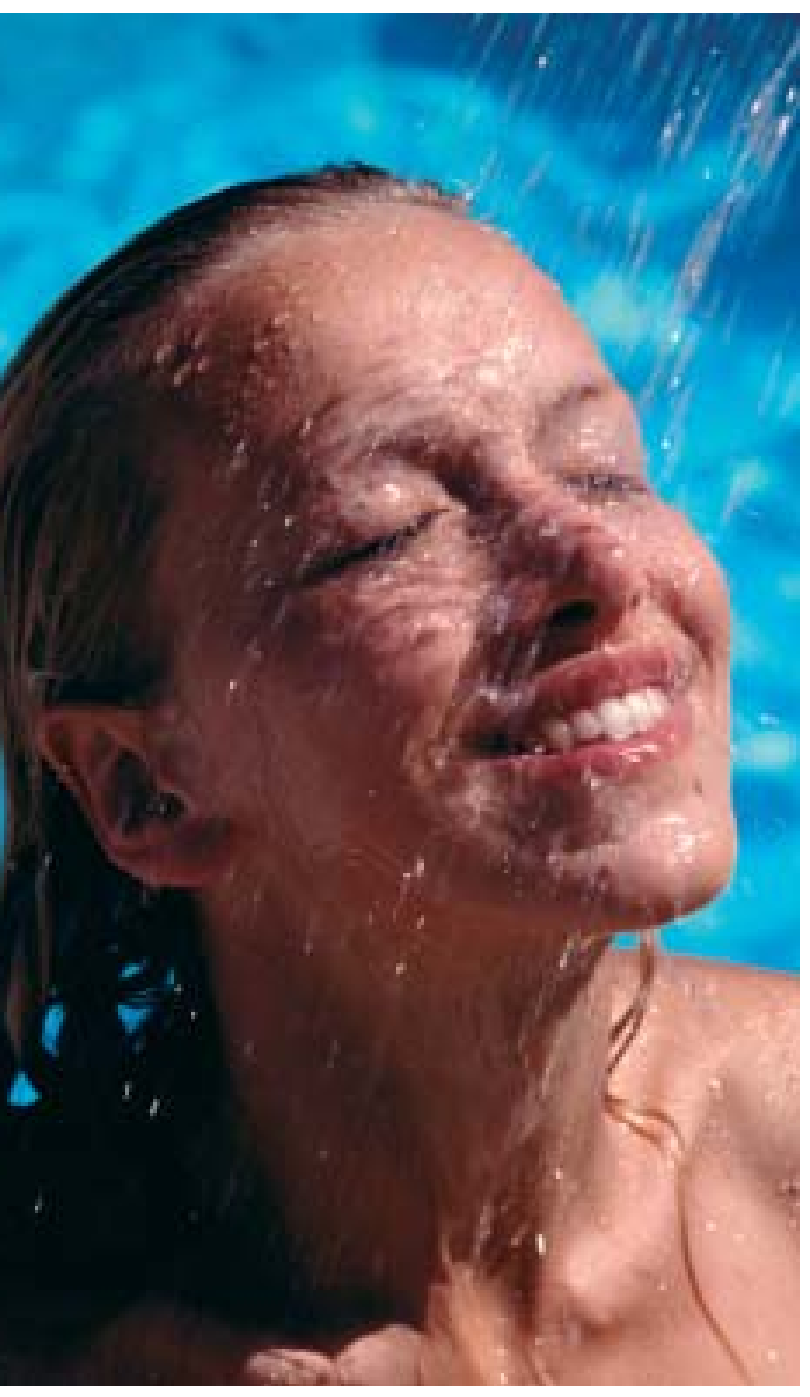

nicht angepasste Kleidung (Uniform) und fehlender Wärmeaustausch durch zu enge Marschformationen ein zusätzlicher Auslösefaktor. Zur Behandlung genügt es, enge Kleider zu öffnen, den Kranken im Schatten flach zu lagern und ihm (nicht zu kaltes!) Mineralwasser zu trinken zu geben.

\section{Hitzschlag}

Stören hohe Temperaturen lange die Wärmeregulation und ist die Wärmeabgabe nicht möglich, so kommt es zum Hitzschlag. Diese Bedingungen wären nach einiger Zeit in einer Sauna gegeben, je höher der Feuchtigkeitsgehalt der Luft, um so eher tritt das Hyperthermiesyndrom auf. Symptome dieses ernsten Schädigungsbildes sind zunächst Kopfschmerzen und Übelkeit. Da der Kreislauf erheblich mehr belastet wird, steigt der Puls, fällt dann der zunächst normale Blutdruck, resultiert Bewusstlosigkeit. Die Körpertemperatur steigt auf über $40{ }^{\circ} \mathrm{C}$, die Haut ist gerötet, oft trocken und heiß. Bewusstseinstrübung und trockene Haut sind typisch für den Hitzschlag und zur Differentialdiagnose wesentlich. Sistiert die Schweißabsonderung als wichtigstes Thermoregulationsmittel, steigt die Temperatur weiter, und es tritt bei ca. 40,5-41,6 ${ }^{\circ} \mathrm{C}$ Bewusstlosigkeit ein und bei $43,5^{\circ} \mathrm{C}$ irreversible Schäden bis hin zum Tod. Als erste Notfallmaßnahme wird der Patient sofort und schnell an einen kühleren Ort gebracht, und der Körper mit (nicht eis-!) kalten Umschlägen auf zirka $38{ }^{\circ} \mathrm{C}$ abgekühlt. Als Notfall ist eine stationäre Überwachung angezeigt. Falls möglich, wird über eine Verweilkanüle bereits mit der Elektrolytsubstitution begonnen, auch eine $\mathrm{O}_{2}$-Gabe ist nützlich.

\section{Hitzekrämpfe}

Selten können Hitzekrämpfe bei schwerer Arbeit in hoher Umgebungstemperatur auftreten, wenn durch Schwitzen mehrere Liter (2-4 Liter) extrazellulärer Flüssigkeit und $\mathrm{NaCl}$ verloren gehen. Muskelschmerzen und -zuckungen bis zu Krämpfen treten auf. Reichliche Zufuhr von Mineralwasser schafft Abhilfe.

\section{Sonnenstich}

Wer sich starker Sonneneinstrahlung ohne Kopfbedeckung aussetzt, kann durch diese unmittelbare Einwirkung einen „Sonnenstich“ bekommen, der mit heftigen Kopfschmerzen, Übelkeit, Fieber, Schwindel, Ohrensausen und orthostatischem Kollaps verbunden ist. Die Hirndrucksteigerung kann in schweren Fällen auch ein Koma und generalisierte Krämpfe auslösen. Zur Therapie wird der leicht erhöht gelagerte Kopf durch feuchte Tücher gekühlt. Der im Urlaub häufige Alkoholgenuss ist in Kombination mit Hitze gefährlich. Die Blutgefäße weiten sich, reagieren schlechter, das Verhalten wird risikoreicher. Säuglinge, Kleinkinder und sehr alte Menschen können ihren Wärmehaushalt schlechter regulieren, sie dekompensieren eher als gesunde Erwachsene. Bei sehr alten Menschen (über 80 Jahre) kommt erschwerend hinzu, dass das Verhalten nicht der Hitze angepasst wird: Falsche Kleidung, das stets zu geringe Durstgefühl, unterschiedliche Organinsuffizienzen (Myokardinsuffizienz) können die körpereigene Thermoregulation erheblich einschränken. Auch durch Medikamente - Diuretika - werden Ältere belastet.

\section{Diarrhoe}

Mehr als zwei ungeformte Stühle täglich mit den wechselnden Allgemeinsymptomen Übelkeit, Erbrechen, Schmerzen, Meteorismus, aber auch ein symptomfreies Intervall sind typisch für eine Diarrhoe. Unter den vielen Ursachen ist die Gastroenteritis häufg, vor allem bei Fieber als Zeichen einer infektiösen Durchfallerkrankung; deren Ursachen sind hauptsächlich Viren (Rotaviren), Bakterien (Toxine) und Parasiten. Die akute Diarrhoe dauert meist nicht länger als einige Tage; sie ist eine selbst-limitierende Krankheit. Anamnese, klinische Untersuchung und die Inspektion des Stuhls ergeben eine vorläufige Diagnose. Sind dabei Blut- und Schleimbeimengungen zu eruieren, ist unter der Verdachtsdiagnose „bakterielle Enteritis“ ein Erregernachweis (Stuhlprobe) zu führen und die Mel- 


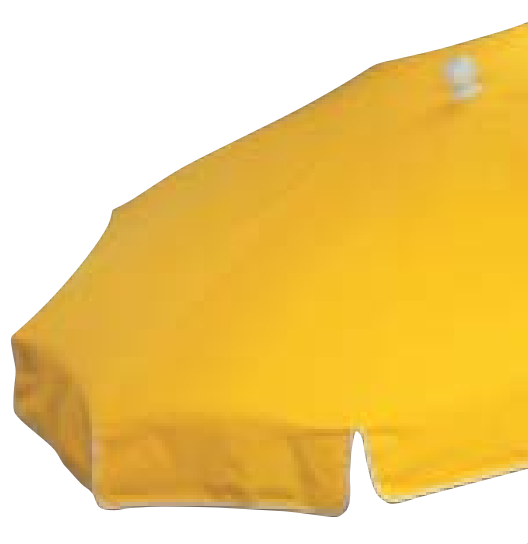

depflicht zu beachten. Die Übertragung auf den Menschen erfolgt durch infizierte Eier, Geflügel und einige andere Lebensmittel. Salmonella typhimurium, ein Serotyp von Salmonella enteritidis, ruft die Salmonellen-Gastroenteritis hervor, eine Form der Lebensmittelvergiftung mit Bauchschmerzen, Fieber, Übelkeit, Erbrechen und Durchfall. Die Inkubationszeit beträgt acht bis 48 Stunden, und die Erkrankung dauert drei bis sieben Tage. Die infektiöse Gastroenteritis (bakterielle Lebensmittelvergiftung) ist eine ansteckende Schleimhautentzündung von Magen (Gastritis) und Dünndarm (Enteritis). Dieser „akute Brechdurchfall“ oder auch Reiseoder Sommerdiarrhoe, hat seine Ursache in verdorbenen Lebensmitteln, an heißen Tagen beispielsweise durch falsche Lagerung (Fleischsalat). Neben Salmonellen kommen unter anderem auch Shigellen und Kolibakterien in Betracht. Die Erkrankung beginnt plötzlich mit hohem Fieber, starkem Erbrechen, Bauchschmerzen, Durchfall mit häufigen dünnen Stühlen, Schleim- und Blutbeimengungen; durch den Flüssigkeitsverlust kann eine Exsikkose (Wadenschmerz!) auftreten. Bei der Enterokolitis fehlt das Erbrechen. Liegt die Ursache des Durchfalls im Dünndarm, ist das Allgemeinbefinden beeinträchtigt, und es treten kolikartige Schmerzen im Oberbauch auf. Vom Kolon stammender Durchfall erzeugt weniger ein Krankheitsgefühl, die „Abdominalkolik“, der Schmerz im Unterbauch, führt dann zur Konsultation. Häufig haben die Kranken auch keine Schmerzen. Sehr alte Menschen sind wegen eines schnelleren Elektrolyt- und Wasserverlustes mit nachfolgender Niereninsuffizienz und Hirnleistungsstörung besonders zu überwachen und ausreichend zu rehydrieren.
Notsituation

gezeigt, da sie lediglich die Funktion des Darmes drosseln. Dadurch werden die Durchfälle zwar seltener, der Krankheitsverlauf aber nicht positiv beeinflusst. Antibiotika verzögern den Heilverlauf, sie sind in unseren Breiten nicht indiziert. Die kolikartigen Schmerzen sind meist von so kurzer Dauer, dass eine spasmolytisch-analgetische Therapie nicht notwendig ist. Dauert die Krankheit länger als drei Tage, muss eine genauere Diagnostik erfolgen. schen Dekompensation zu rechnen. Kleinkinder werden mit einem Glukose-Elektrolyt-Gemisch (z.B. Oralpädon ${ }^{\circledR}$ ) rehydriert. Kinder unter zwei Jahren reagieren auf Störungen ihres Elektrolyt- und Wasserhaushaltes besonders empfindlich. Sie sind sorgfältig zu therapieren und zu überwachen, Kinder unter einem Jahr sind bei gleichzeitigem Durchfall und Erbrechen so gefährdet, dass stationär rehydriert werden muss. Trotz der unmittelbaren Gefahr durch Wasser- und Elektrolytverluste heilt die Entzündung ohne spezifische Medikamente in zwei bis sieben Tagen aus. Allgemein steht die Therapie auf drei Säulen: Diät, Verhalten und Medikamente. Erwachsene schonen ihren Verdauungstrakt bis zur Besserung, indem sie nichts bis wenig leicht Verdauliches essen (Zwieback, Salzstangen, geriebener Apfel, Banane), Kinder bekommen „Heilnahrung“, zusätzlich reichlich Kamillentee und Mineralwasser. Als Elektrolytersatz mit Glucose gibt man zum Beispiel Elotrans ${ }^{\circledR}$, bei Kindern Oralpädon ${ }^{\circledR}$. Wenn oral zugeführte Flüssigkeit erbrochen wird, ist eine Infusionstherapie zu erwägen (Wasser- und Elektrolytersatz wie Ringer ${ }^{\circledR}$, Tutofusin ${ }^{\circledR}$ etc.). Da Cola von allen Kindern gerne getrunken wird und damit der Zwang zu trinken entfällt, ist das Gemisch zwei Teile Mineralwasser und ein Teil Cola zu empfehlen. Cola enthält Bikarbonat, Kohlenhydrate und Natrium, das enthaltene Koffein begrenzt die Dosis. Auch im Gemisch bleibt der Kalium-Gehalt zu niedrig. Säuglinge brauchen 150 ml Flüssigkeit pro kg Körpergewicht und 24 Stunden. Von den Antidiarrhoika kann Kohle (Kohle-Compretten) einen Nutzen haben. Andere Mittel z.B. Loperamid - sind nur selten an-

\section{Summary}

The energy of the sun's rays can damage the human organism by causing skin, eye and immune system reactions. Elevated levels of ozone in the summer can result in inflammation of mucous membranes giving rise to conjunctivitis, pharyngitis, laryngitis and tracheitis, sometimes coupled with headache. In general, the sequelae of a disturbance of thermal homeostasis is referred to as heat injury, and can cover a range of harmful effects. In the case of heat exhaustion, the organism reacts with various signs of shock. If high temperatures disturb thermal regulation over longer periods, heat stroke may ensue. Strong solar radiation to the uncovered head can result in sunstroke, which is associated with severe headaches, nausea, fever, dizzy spells, and orthostatic collapse. Acute diarrhea and vomiting or travellers or summer diarrhea on hot days is caused by foods that have gone off through inappropriate storage.

\section{Literatur}

Literatur beim Verfasser

\section{Anschrift des Verfassers}

Dr. med. Ulrich Rendenbach

Marktstraße 7

37115 Duderstadt 